\title{
Freeze-Drying Formulations Increased the Adenovirus and Poxvirus Vaccine Storage Times and Antigen Stabilities
}

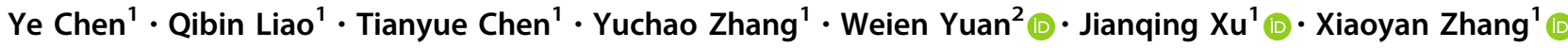

Received: 19 April 2020 / Accepted: 9 June 2020/Published online: 21 July 2020

(C) Wuhan Institute of Virology, CAS 2020

\begin{abstract}
Successful vaccines induce specific immune responses and protect against various viral and bacterial infections. Noninactivated vaccines, especially viral vector vaccines such as adenovirus and poxvirus vaccines, dominate the vaccine market because their viral particles are able to replicate and proliferate in vivo and produce lasting immunity in a manner similar to natural infection. One challenge of human and livestock vaccination is vaccine stability related to the antigenicity and infectivity. Freeze-drying is the typical method to maintain virus vaccine stability, while cold chain transportation is required for temperatures about $2{ }^{\circ} \mathrm{C}-8{ }^{\circ} \mathrm{C}$. The financial and technological resource requirements hinder vaccine distribution in underdeveloped areas. In this study, we developed a freeze-drying formula consisting of bovine serum albumin (BSA), L-glutamic acid (L-Glu), polyethylene glycol (PEG), and dextran (DEX) to improve the thermal stability and activity of viral vaccines, including vaccinia recombinant vaccine (rTTV-OVA) and adenovirus vaccine (Ad5-ENV). We compared a panel of five different formulations (PEG: DEX: BSA: L-GLU = 50:9:0:0(\#1), 50:5:4:0(\#2), 50:10:9:0(\#3), 50:0:0:9(\#4), and 50:1:0:8(\#5), respectively) and optimized the freeze-drying formula for rTTV-OVA and Ad5-ENV. We found that the freeze-drying formulations \#2 and \#3 could maintain rTTV-OVA infectivity at temperatures of $4{ }^{\circ} \mathrm{C}$ and $25^{\circ} \mathrm{C}$ and that rTTV-OVA immunogenicity was retained during lyophilization. However, formulations \#4 and \#5 maintained Ad5-ENV infectivity under the same conditions, and Ad5-ENV immunogenicity had maximum retention with freeze-drying formulation \#4. In summary, we developed new freeze-drying formulations that increased virus vaccine storage times and retained immunogenicity at an ambient temperature.
\end{abstract}

Keywords Adenovirus vaccine $\cdot$ Poxvirus vaccine $\cdot$ Freeze-drying formulation $\cdot$ Stability $\cdot$ Immunogenicity

Xiaoyan Zhang

zhangxiaoyan@ @hphc.org.cn

$\triangle$ Jianqing $\mathrm{Xu}$

xujianqing@shphc.org.cn

$\triangle$ Weien Yuan

yuanweien@126.com

1 Shanghai Public Health Clinical Center and Institutes of Biomedical Sciences, Fudan University, Shanghai 201508, China

2 Engineering Research Center of Cell and Therapeutic Antibody, Ministry of Education, and School of Pharmacy, Shanghai Jiao Tong University, Shanghai 200000, China

\section{Introduction}

Vaccination is the most cost-effective strategy for the prevention and control of infectious diseases (Jang and Seong 2013; Reid et al. 2019). With the development of animal husbandry and the prosperity of the pet market, the number of vaccines administered to animals is significantly increasing (Baron et al. 2018). Noninactivated vaccines, including recombinant viral vector vaccines, attenuated live vaccines, and genetically engineered vaccines (protein subunit vaccines and nucleic acid vaccines), account for a large market share (Melief et al. 2015; Meri et al. 2008).

The activity of the noninactivated vaccines depends on their immunogenicity and delivery strategies, as well as on the stability of the vaccines during storage and transportation (Peetermans 1996; Walters et al. 2014). Several factors, including ambient temperature, $\mathrm{pH}$, freezing effect 
and dehydration, might result in lipid damage, protein damage, and nucleic acid degradation (Abdul-Fattah et al. 2007; Chen et al. 2010); ultimately, these factors may affect the stability of the vaccines (Kasper and Friess 2011). Therefore, most of the noninactivated vaccines are directly stored at temperatures under $4{ }^{\circ} \mathrm{C}$ or distributed through cold chain transportation after lyophilization, which significantly increases the cost and greatly restricts the accessibility and feasibility of the vaccines, especially in underdeveloped areas (Briggs and Ilett 1993; Hansen et al. 2015). Determining how to keep the vaccines stable at ambient temperature, reduce the loss of vaccine activity and control the economic cost of vaccine production and distribution are major challenges for the vaccine industry (Madan et al. 2018).

Freeze-drying protectants are complex reagents that prevent active component denaturation in vaccines during freeze-drying processes (Hansen et al. 2015). Under the condition of cold chain logistics, the relatively effective and common formula of cryoprotectants includes monosaccharide, polyalcohol, amino acids, salts and cryoprotectant derivatives (Basu and Domb 2018; Borde et al. 2011; Ohtake et al. 2011) such as sucrose, phosphate, glutamic acid and albumin (SPGA); lactose, gelatin, sorbitol and HEPES buffer (LGS); potassium phosphate buffer, hydrolysate gelatin and sorbitol (BUGS); and lactose hydrolysate and sucrose (LS) (Chen et al. 2012; Prabhu et al. 2014; Zheng et al. 2019). However, the thermostability and protection of these freeze-drying protectants are unsatisfactory (Siow et al. 2016). In particular, viral vaccines in cryoprotection medium are not stable at temperatures above $8{ }^{\circ} \mathrm{C}$ (Hansen et al. 2015; Orr et al. 2014). The loss of activity increases as the temperature rises. Furthermore, viral vaccines require cold chain logistics (processing, storage and distribution) (Orr et al. 2014). It is necessary to further improve their thermal stability and protective effect, especially in ambient temperatures.

Polyethylene glycol (PEG), dextran (DEX), and bovine serum albumin (BSA) are often individually used for the freeze-drying protection of clinical drugs, lactic acid bacteria, and microbes (Mody et al. 2014). PEG can reduce the damage to the virus through dehydration. DEX, a type of oligosaccharide, can protect viral activity from damage (Kraan et al. 2014). BSA, a kind of enzyme stabilizer, can prevent enzyme decomposition and denaturation. In addition, L-glutamic acid, with both acidic and basic amino acid ions, can buffer the $\mathrm{pH}$ changes in the freeze-drying process and can protect the vaccine activity. Use of L-glutamate has not been reported as a freeze-drying protective agent. The materials described here might be components of novel cryoprotectants.

In this study, we selected recombinant viral vector vaccines as representative noninactivated vaccines, including enveloped poxvirus recombinant vaccine and nonenveloped adenovirus recombinant vaccine, to explore the effect of the newly constructed vaccine protectant on the stability and immunogenicity of noninactivated vaccines under cold and ambient temperatures $\left(4{ }^{\circ} \mathrm{C}\right.$ and $\left.25^{\circ} \mathrm{C}\right)$. We hope to provide proof of the new freeze-drying formula to protect the activity of the noninactivated vaccines for animal vaccinations.

\section{Materials and Methods}

\section{Vaccine Strains and Cell Lines}

DNA vaccines expressing OVA and ENV were constructed by cloning the target genes into $\mathrm{pSV}-1.0$ vector and all DNA vaccines used in this study were prepared by using the Endo-free Plasmid Giga Kit (Qiagen, Cat\#12391). Recombinant Tiantan vaccinia encoding OVA (rTTVOVA) was constructed in our previous work (Qiu et al. 2014). The virus was propagated in Vero cells (ATCC CCL-81, passage 135e160). The cells were cultivated in Dulbecco's modified Eagle's medium (DMEM, Corning) containing $10 \%$ fetal bovine serum (FBS, BI) with $1 \%$ penicillin/streptomycin solution (P/S, ScienCell), and DMEM with $2 \%$ FBS (BI) was used for maintenance. Adenovirus type 5 encoding ENV (Ad5-ENV) was constructed and amplified by Shanghai Seven Sea Pharmtech Biotechnology Co., Ltd.

\section{Animal Study}

The mice used in this study were 8-10 week-old female C57BL/6 mice, which were purchased from the B\&K Universal Group Limited (Shanghai, China) and maintained under specific pathogen-free (SPF) conditions at the animal facilities of Shanghai Public Health Clinical Center (Shanghai, China). For each experimental group, there were at least six mice analyzed at each collection time point. For vaccination experiments, mice were intramuscular (i.m) inoculated with either $100 \mathrm{ng}$ DNA vaccine (pSV1.0-OVA or pSV1.0-ENV) or empty vector pSV1.0 as control. The viral vaccine including $10^{7}$ PFUs of rTTVOVA or $10^{8}$ TCID50 of Ad5-ENV in a volume of $100 \mu \mathrm{L}$ was also inoculated intranasally. The mice were immunized twice with DNA vaccine followed by the viral vaccine at 2 -week intervals. After vaccination, mice were monitored daily for clinical symptoms and survival. For immunoassay, immunized mice were sacrificed on day 14 after the last immunization for collection of peripheral blood and spleen tissues from which mononuclear cells (PBMCs) and single-splenocytes were obtained and analyzed, respectively. The animal experiments were 
performed in accordance with Home Office guidelines and were approved by the Institutional Animal Care and Use Committee (IACUC) of Shanghai Public Health Center.

\section{Formulations of the Freeze-Drying Protectant and Lyophilization}

We used deionized water as the solvent and prepared five freeze-drying protectants consisting of PEG, DEX, BSA and L-Glu. The detailed formulas, including the weight/ volume ratio of each ingredient, are indicated in Table 1. The final concentration of the protectant was $1 \mathrm{mg} / \mathrm{mL}$. Then, the constructed protectant was mixed with the different viral vaccines in $1 \mathrm{~mL}$, and the protectant-to-vaccine volume ratio was 49:1. The viruses were then stored at $-80{ }^{\circ} \mathrm{C}$ overnight and were lyophilized at $-50{ }^{\circ} \mathrm{C}$ and $0.05 \mathrm{mbar}$ for $24 \mathrm{~h}$. Batches of freeze-dried vaccines were stored for 7,14 and 28 days at $4{ }^{\circ} \mathrm{C}$ and $25^{\circ} \mathrm{C}$, respectively.

\section{Titration of Viral Vaccines}

After storage at the indicated temperature and time as mentioned above, the samples containing the rTTV-OVA and Ad5-ENV vaccines were separately rehydrated and serial diluted by tenfold in DMEM with 10\% FBS.

Vero cells were cocultured with different concentrations of rTTV-OVA in duplicate in 24 -well plates $(500 \mu \mathrm{L} /$ well $)$ within maintenance medium at $37^{\circ} \mathrm{C}$ for $48 \mathrm{~h}$. Thereafter, a titrating mixture consisting of $2 \times$ DMEM and lowmelting-point agarose (1:1) with X-gal $(200 \mu \mathrm{g} / \mathrm{mL})$ was added to the culture ( $300 \mu \mathrm{L} /$ well). After the mixture in the plate solidified at $4{ }^{\circ} \mathrm{C}$, the plate was continuously incubated at $37^{\circ} \mathrm{C}$ for $4 \mathrm{~h}$. The titer of the vaccine was determined by counting the blue viral plaques per well.

On the other hand, Ad5-ENV was titrated with different cell culture models. Different concentrations of Ad5-ENV were cocultured with $293 \mathrm{~A}$ cells in ten replicates within 96-well plates $(100 \mu \mathrm{L} /$ well $)$ at $37{ }^{\circ} \mathrm{C}$ for 10 days. The titer of Ad5-ENV was determined by observing the cell cytopathic effect (CPE) per well according to the formula: $T=10^{1+d(S-0.5)} / 0.1 \mathrm{~mL}\left(d\right.$ : $\log _{10}$ dilution, $S$ : the number of wells that have CPE in ten replicates).

\section{Enzyme-Linked Immunosorbent Spot (ELISPOT) Assay}

The vTTV-OVA- or Ad5-ENV-induced immune responses of mice were investigated using IFN- $\gamma$ ELISPOT assays (BD Bioscience, Cat\# 551083). A 96-well ELISPOT plate was coated with an anti-mouse IFN- $\gamma$ monoclonal antibody (1:250 diluted with coating buffer, $100 \mu \mathrm{L} /$ well) at $4{ }^{\circ} \mathrm{C}$ overnight. Then, the mouse splenocytes collected above $\left(2 \times 10^{5}\right)$ and the peptides (OVA peptide : OVA ${ }_{(257-264)}$ and OVA (323-339); ENV peptide pool: $\left.\mathrm{ENV}_{(\mathrm{B} \text { strain, RL 42) }}\right)$ $(10 \mu \mathrm{g} / \mathrm{mL})$ were added into each well $(50 \mu \mathrm{L} /$ well) (OVA peptide were synthesized by Shanghai Science Peptide Biological Technology Co. Ltd and ENV peptide pool were synthesized by GL Biochem (Shanghai) Ltd). The response induced by OVA was detected using peptide OVA (257-264) and OVA (323-339), and the response induced by ENV was detected using the ENV peptide pool (B strain, RL 42). The plate was incubated at $37^{\circ} \mathrm{C}$ for $20 \mathrm{~h}$ in a fully humidified atmosphere with $5 \% \mathrm{CO}_{2}$ and was subjected to the detection of IFN- $\gamma$ release using an automated ELISPOT plate reader (ChampSpot III Elispot Reader, Saizhi, Beijing, China). Each spot represented an IFN- $\gamma$-producing splenocyte, and more than 50 spots $/ 10^{6}$ cells were determined to be positive.

\section{Intracellular Cytokine Staining (ICS)}

Freshly isolated splenocytes or lymphocytes were plated into round-bottom 96 -well plates $\left(2 \times 10^{6}\right.$ cells per well $)$ and stimulated with $5 \mu \mathrm{g} / \mathrm{mL}$ OVA peptide: $\mathrm{OVA}_{(257-264)}$ and OVA $_{(323-339)}$ and ENV peptide pool: $\mathrm{ENV}_{(\mathrm{B} \text { strain, RL 42) }} .1 \mathrm{~h}$ later, brefeldin A (Cell Signaling Technology Cat\#9972S) and monesin (Sigma Cat\#1445481) were added to each well at final concentrations of $1 \mu \mathrm{g} / \mathrm{mL}$ and $1 \mu \mathrm{mol} / \mathrm{L}$. Then $7 \mathrm{~h}$ later, the stimulation were stopped by washing the plates

Table 1 Compositions of the freeze-drying formulations.

\begin{tabular}{llllll}
\hline Ingredients & $\begin{array}{l}\text { Formulation } 1 \\
(\mathrm{FR} 1, \mathrm{w} / \mathrm{v})\end{array}$ & $\begin{array}{l}\text { Formulation } 2 \\
(\mathrm{FR} 2, \mathrm{w} / \mathrm{v})\end{array}$ & $\begin{array}{l}\text { Formulation } 3 \\
(\mathrm{FR} 3, \mathrm{w} / \mathrm{v})\end{array}$ & $\begin{array}{l}\text { Formulation } 4 \\
(\mathrm{FR} 4, \mathrm{w} / \mathrm{v})\end{array}$ & $\begin{array}{l}\text { Formulation 5 } \\
(\mathrm{FR} 5, \text { w/v) }\end{array}$ \\
\hline PEG & 50 & 50 & 50 & 50 & 50 \\
DEX & 9 & 5 & 10 & - & 1 \\
BSA & - & 4 & 9 & - & - \\
L-Glu & - & - & - & 9 & 8 \\
\hline
\end{tabular}

PEG: polyethylene glycol; DEX: dextran; BSA: bovine serum albumin; L-Glu: L-glutamic acid.

“_" means not included. 
with R10 medium and 4 cell surface markers (CD3, CD8, CD44, and CD62L) were stained on ice with uorescein labeled antibodies, including PerCP-Cy5.5-labeled antimouse CD3 (Clone: 17A2, Biolegend Cat\# 100218), Pacic Blue labeled anti-mouse CD8 (Clone: 53-6.7, Biolegend Cat\# 100725), FITC-labeled anti-mouse CD44 (Clone: IM7, eBioscience Cat\# 11-0441-81), and APC-eFluor780-labeled anti-mouse CD62L (Clone: MEL-14, eBioscience Cat\# 47-0621-80). Next, the cells were fixed and permeablized, and intracellular IFN- $\gamma$ was stained with PE-conjugated antimouse IFN- $\gamma$ (Clone: XMG1.2, Biolegend Cat\# 505808). Stained samples were measured using BD FACS Aria I. Data analysis was done by using FlowJo X software (Tree Star, Inc).

\section{Antibody Titration}

ELISA was used to assess the serum antibody titers against OVA and ENV. Each well of a 96-well EIA/RIA plate was coated with $100 \mu \mathrm{L}$ of OVA protein $(1 \mu \mathrm{g} / \mathrm{mL})$ or ENV protein $(1 \mu \mathrm{g} / \mathrm{mL})$ at $4{ }^{\circ} \mathrm{C}$ overnight and was blocked with $200 \mu \mathrm{L} \mathrm{10 \%} \mathrm{FBS.} \mathrm{The} \mathrm{immunized} \mathrm{mice} \mathrm{sera} \mathrm{were} \mathrm{diluted}$ twofold, and $100 \mu \mathrm{L} / w e l l$ was added to the plate. The goat anti-mouse $\operatorname{IgG}(1: 2000)$ was used to detect the antibody against OVA or ENV in mouse serum. Mouse serum antibody titers were assessed with the microplate reader (absorbance at $450-570 \mathrm{~nm}$ ) and calculated by the correction number larger than $2 \times$ (negative mean $+\mathrm{SD}$ ).

\section{Statistical Analysis}

All data were expressed as the mean $\pm \mathrm{SD}$ of each group, and the difference comparison between two groups was conducted by unpaired, two-tailed Student's $t$-tests. The difference was considered statistically significant when $P \quad$ values were $<0.05 \quad(* * * P<0.001 ; \quad * * P<0.01$; $* P<0.05)$.

\section{Results}

\section{Efficacy of Different Formulations for Two Kind of Vaccines Under Different Conditions}

To test the protection of the formulations from freezedrying damage, the titration of vaccine rTTV-OVA or Ad5ENV was performed after storage at the indicated temperature and time as mentioned above.

The titer of poxvirus vaccine rTTV-OVA post lyophilization in formulation \#3 (FR3) was the highest compared to those in PBS $(P=0.0058)$ and other formulations (Fig. 1A). Then, we explored the protection of freeze-drying formulations under cold chain conditions.
After storage at $4{ }^{\circ} \mathrm{C}$ for 7 to 28 days, the titers of rTTVOVA in FR2 $\left(4.37 \times 10^{7} \mathrm{PFUs} / \mathrm{mL}\right)$ and FR3 $\left(4.35 \times 10^{7}\right.$ PFUs $/ \mathrm{mL}$ ) were higher than those in other formulations 28 days post storage $(P<0.001)$ (Fig. 1B). Therefore, FR2 and FR3 were used as the optimal formulations for rTTV-OVA stored at $4{ }^{\circ} \mathrm{C}$ in the next study.

After 28 days of storage at $25{ }^{\circ} \mathrm{C}$, the titers of rTTVOVA in FR2 and FR3 were still above $1 \times 10^{6} \mathrm{PFUs} / \mathrm{mL}$, while the titers of other groups were almost unable to be detected (Fig. 1C). Therefore, the freeze-drying formulations \#2 and \#3 maintained the infectivity of rTTV-OVA when stored at $4{ }^{\circ} \mathrm{C}$ and at the ambient temperature.

However, FR2 and FR3 did not protect the adenovirus vaccine Ad5-ENV post lyophilization (data not shown). In contrast, the titers of Ad5-ENV maintained in FR4 $\left(4.28 \times 10^{8} \quad\right.$ TCID50/mL, $\left.\quad P<0.001\right) \quad$ and $\quad$ FR5 $\left(3.93 \times 10^{8}\right.$ TCID50/mL, $\left.P<0.001\right)$ were effectively protected (Fig. 2A). Ad5-ENV was treated similarly to rTTV-OVA. After storage at $4{ }^{\circ} \mathrm{C}$ for up to 28 days, the titer of Ad5-ENV was reduced slightly ( 0.3 to $0.5 \log$ of TCID50/mL) when it was stored in FR4, FR5 and FR1, but those stored in PBS were reduced significantly (3 log of TCID50/mL) (Fig. 2B). After stored at $25^{\circ} \mathrm{C}$ for 28 days, the titer of Ad5-ENV in FR4 and FR5 decreased 0.6 to 1 $\log$ of TCID50/mL $\left(5.73 \times 10^{7}\right.$ TCID50/mL and $1 \times 10^{7}$ TCID50/mL, respectively), while those in other groups were almost unable to be detected (Fig. 2C). The freezedrying formulations \#4 and \#5 maintained the infectivity of Ad5-ENV at storage temperatures of $4{ }^{\circ} \mathrm{C}$ and the ambient temperature.

\section{Immunogenicity and Efficacy of the Viral Vaccines in Different Formulations}

According to the previous screening results, FR2 and FR3 were used to protect rTTV-OVA, and FR4 and FR5 were used to protect Ad5-ENV in the following immunogenicity study.

\section{Immunogenicity and Efficacy of rTTV-OVA Under the Protection of FR2 and FR3}

To determine whether the newly prepared freeze-drying formulations FR2 and FR3 could protect the immunogenicity of the recombinant poxvirus vaccine rTTV-OVA, we immunized C57BL/6 mice with a prime/boost strategy (Fig. 3A). Briefly, four groups of mice were immunized three times with 14 days interval. The empty DNA vector pSV1.0 was used for all three inoculations in the mock group. In the other three experimental groups, the first two inoculations of priming antigens were all DNA vaccines (pSV1.0-OVA), but the boosting antigens of the 3rd inoculation was different and consisted of rTTV-OVA without 
A

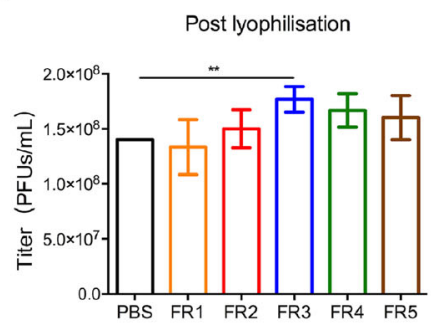

B

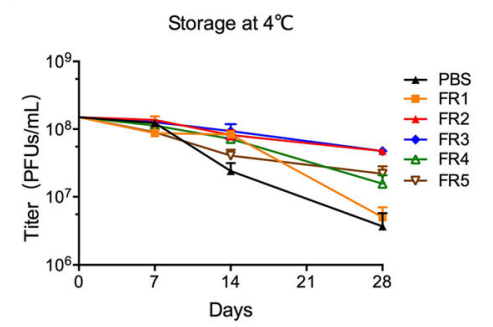

C

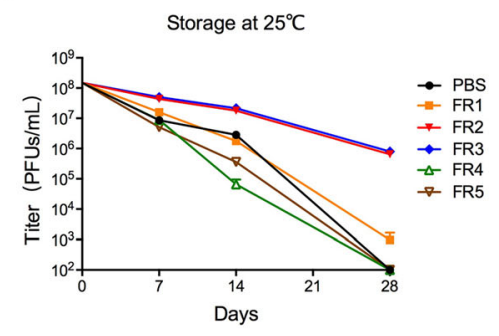

Fig. 1 Freeze-drying formulations \#2 and \#3 effectively protected the activity of poxvirus vaccine. The infectivity of recombinant poxvirus vaccines (rTTV-OVA) protected by six formulations were detected post lyophilization and in different storage conditions. The titer is detected by counting the blue viral plaques per well. A Titers of poxvirus in six formulations post lyophilization. The titer of FR3 was slightly higher than that of the other formulations $(* * P<0.01)$. BC The protected rTTV-OVAs were stored at $4{ }^{\circ} \mathrm{C}(\mathbf{B})$ or $25{ }^{\circ} \mathrm{C}(\mathbf{C})$ for $7 / 14 / 28$ days post lyophilization. The titers of vaccines in FR2 and FR3 are higher than that in any other formulation.
A

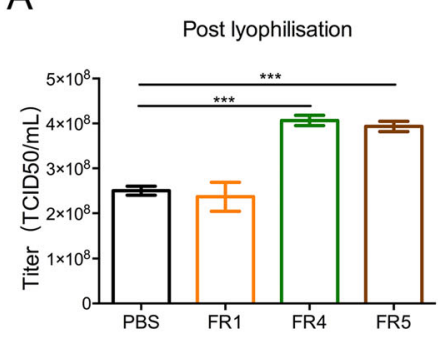

B

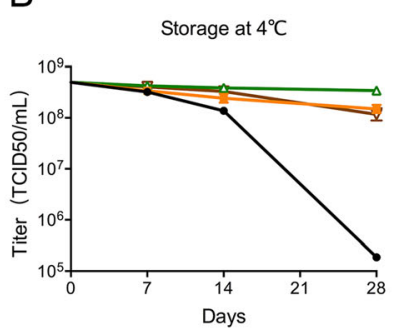

C

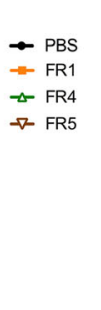

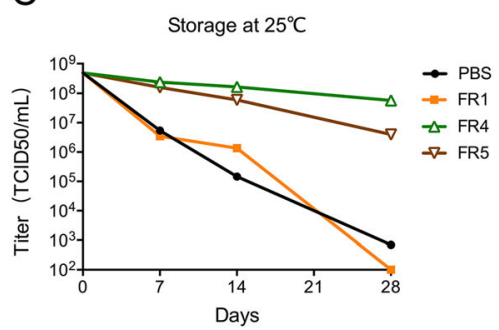

Fig. 2 Freeze-drying formulations \#4 and \#5 effectively protected adenovirus vaccine activity. The infectivity of recombinant adenovirus vaccines (Ad5-ENV) protected by different formulations was detected post lyophilization. The titer is detected by 293A cells. A Image showing titers of adenovirus in four formulations post

lyophilization or with lyophilization in FR2 or FR3, respectively.

Two weeks after the last vaccination with rTTV-OVA, the mice were euthanized, and the splenocytes were collected for immune response detection.

First, the IFN- $\gamma$ response was detected using ELISPOT, and there was no significant difference in the number of spot-forming cells (SFCs) $/ 10^{6}$ splenocytes in the lyophilized group FR2 $(202.20 \pm 48.08)$ or group FR3 $(246.00 \pm 77.25)$ compared to the nonfreeze-drying group (NF) $(246.00 \pm 77.25)$ (Fig. 3B). The same phenomenon was also observed when the IFN- $\gamma$ response in $\mathrm{CD} 8+\mathrm{T}$ cells was detected using intracellular cytokine staining (ICS) (Fig. 3C). Moreover, the humoral immune response was investigated using antibody titration. The titer of the binding antibody in non-freeze-drying group (NF) was $3.605 \pm 0.142$, which was comparable to those in groups FR2 (3.755 \pm 0.112$)$ and FR3 (3.66 \pm 0.150$)$ (Fig. 3D).

These results suggested that FR2 and FR3 could effectively protect the immunogenicity of the poxvirus vaccine during lyophilization, and the protected vaccine could activate cellular and humoral immunity comparable to those induced by the fresh vaccine. lyophilization. The titers of vaccines in FR4 and FR5 were higher than those in PBS $(* * * P<0.001)$. B-C The protection of Ad5-ENV stored at $4{ }^{\circ} \mathrm{C}(\mathbf{B})$ or $25{ }^{\circ} \mathrm{C}$ (C) for $7 / 14 / 28$ days post lyophilization. The titers of vaccines in FR4 and FR5 are higher than that in FR1.

\section{Immunogenicity and Efficacy of Ad5-ENV Under the Protection of FR4 and FR5}

The protective effect of the freeze-drying formulations FR4 and FR5 on the immunogenicity of the recombinant adenovirus vaccine Ad5-ENV was investigated using C57BL/6 mice with a prime/boost strategy (Fig. 4A) similar to that used for the recombinant poxvirus vaccine rTTV-OVA, except the boosting antigens were Ad5-ENV without lyophilization or Ad5-ENV with lyophilization in FR4 or FR5, respectively.

The immune responses were also detected using ELISPOT and ICS. There was no significant difference in SFCs $/ 10^{6}$ splenocytes observed between the Ad5-ENV lyophilized groups FR4 $(463.82 \pm 102.01)$ and FR5 $(452.92 \pm 246.67)$ and the Ad5-ENV nonlyophilized group NF $(425.00 \pm 218.79)$ (Fig. $4 \mathrm{~B})$. The same phenomenon was also observed in the IFN- $\gamma$ response in CD8+ T cells detected using ICS (Fig. 4C).

In addition, the humoral immune response induced by ENV was investigated using antibody titration. The titer of the binding antibody induced by Ad5-ENV in group NF and group FR4 was similar $(3.555 \pm 0.112$ vs $3.625 \pm 0.147)$, while the titer in group FR5 


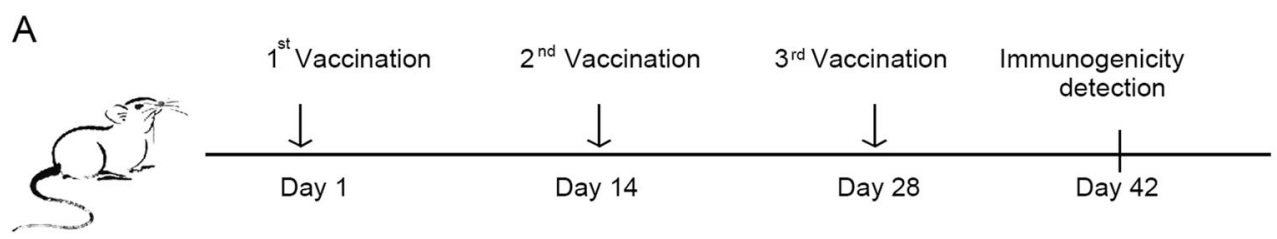

\begin{tabular}{llll}
\hline Groups & $1^{\text {st }}$ Vaccination & $2^{\text {nd }}$ Vaccination & $3^{\text {rd }}$ Vaccination \\
\hline Mock & pSV1.0 & pSV1.0 & pSV1.0 \\
NF & pSV1.0-OVA & pSV1.0-OVA & Nonfreeze-drying rTTV-OVA \\
FR2 & pSV1.0-OVA & pSV1.0-OVA & Formulation2 +rTTV-OVA \\
FR3 & pSV1.0-OVA & pSV1.0-OVA & Formulation3 +rTTV-OVA \\
\hline
\end{tabular}
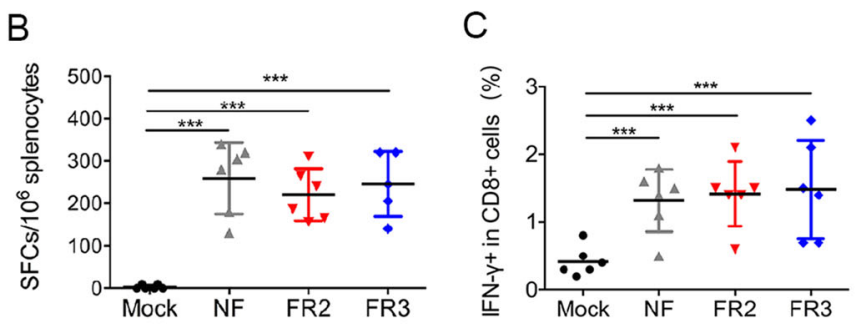

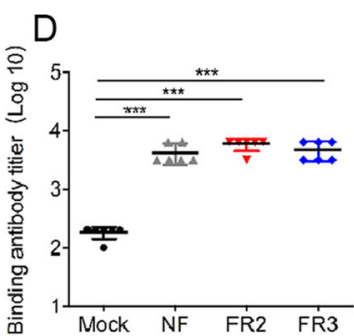

Fig. 3 Freeze-drying formulations \#2 and \#3 protect the immunogenicity of poxvirus vaccine from being damaged due to lyophilization. A Vaccination schedule in C57BL/6 mice. Three different groups were designed using the same two inoculations of the DNA vaccine and different treatments of recombinant poxvirus vaccine. Each DNA vaccine (pSV1.0-OVA $100 \mathrm{ng}$ per mouse) and poxvirus vaccine (rTTV-OVA $10^{7}$ PFUs per mouse) were administered into mice via intramuscular injection at 2 -week intervals. Two weeks after

(3.405 \pm 0.141$)$ was comparable, although slightly decreased (Fig. 4D).

These data implied that FR4 and FR5 could protect the immunogenicity of the recombinant adenovirus vaccine during lyophilization, and the protected vaccine could activate comparable cellular and humoral immunity to those induced by the fresh vaccine.

\section{Discussion}

Previous investigations on vaccine storage conditions were focused on improving the effect of cold chain processing and transportation that consumes more resources (Shin et al. 2018). We designed novel formulations, which could preserve the activity and stability of viral vaccines such as poxvirus vaccine and adenovirus vaccine, during storage at an ambient temperature. Particularly, with the protection of FR4, the titer of the adenovirus vaccine stored at $25^{\circ} \mathrm{C}$ for 28 days only decreased $0.6 \log$. Compared with Ad5 protected in only PEG, we used FR4 (PEG+L-Glu) to the last vaccination, we detected immunogenicity of viral vaccines. B-C The protection of antigen-specific $\mathrm{T}$ cell responses was detected by ELISPOT (B) and intracellular cytokine staining (C). Mouse splenocytes were stimulated by peptide $\operatorname{OVA}_{(257-264)}$ and $\mathrm{OVA}_{(323-339)}$. D The protection of specific binding antibodies was measured by ELISA at the same time. Data are the mean of each cohort $(N=6)$, and error bars indicate SD $(* * * P<0.001)$.

lengthen the storage time by 4 times while the titer value declined by $0.6 \mathrm{log}$. Improving the protection of vaccine at $25{ }^{\circ} \mathrm{C}$ could break the financial and technological barriers to widespread vaccine distribution.

Four ingredients were added to the freeze-drying protectant and were PEG, DEX, BSA and L-Glu. PEG can reduce the damage of the virus through a dehydration effect. DEX, a type of oligosaccharide, can protect viral activity from damage. BSA, a kind of enzyme stabilizer, can prevent enzyme decomposition and denaturation. The materials described here might be components of novel cryoprotectants. L-glutamate has not been reported as a freeze-drying protective agent. However, Glu has both acidic and basic amino acid ions, can buffer the $\mathrm{pH}$ changes in the freeze-drying process and can protect the activity of the vaccines. Different kinds of viral vaccines had their own optimized formulation. The poxvirus vaccine has an entire envelope structure, but the adenovirus vaccine has non-envelope structure. An ingredient shared by FR4 and FR5, but not present in other formulations, is L-Glu. Therefore, we speculate that the protective effect of FR4/ 
A

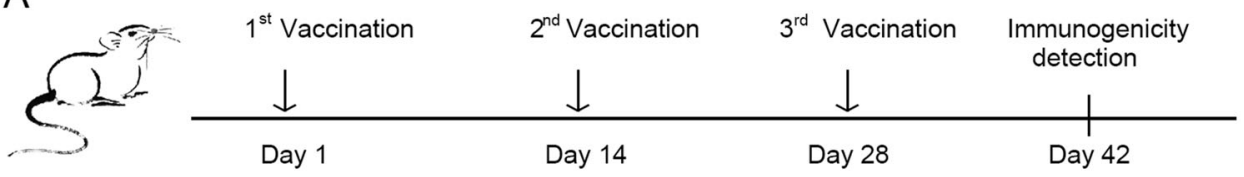

\begin{tabular}{llll}
\hline Groups & $1^{\text {st }}$ Vaccination & $2^{\text {nd } V a c c i n a t i o n ~}$ & $3^{\text {rd } V a c c i n a t i o n ~}$ \\
\hline Mock & $p S V 1.0$ & $p S V 1.0$ & $p S V 1.0$ \\
NF & $p S V 1.0-E N V$ & $p S V 1.0-E N V$ & Nonfreeze-drying Ad5-ENV \\
FR4 & $p S V 1.0-E N V$ & $p S V 1.0-E N V$ & Formulation4+Ad5-ENV \\
FR5 & $p S V 1.0-E N V$ & $p S V 1.0-E N V$ & Formulation5+Ad5-ENV \\
\hline
\end{tabular}
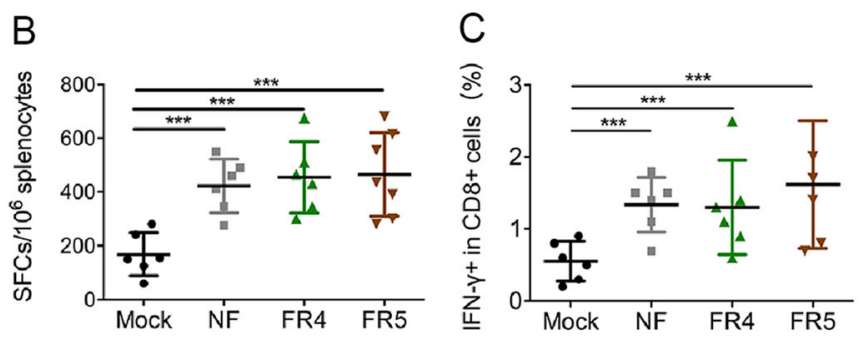

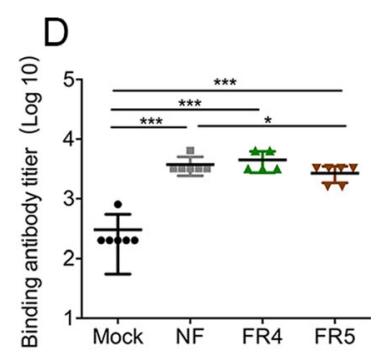

Fig. 4 Freeze-drying formulations \#4 and \#5 protect the immunogenicity of adenovirus vaccine from being damaged due to lyophilization. A Vaccination schedule in C57BL/6 mice. Three different groups were designed using the same two inoculations of the DNA vaccine and different treatments of recombinant adenovirus vaccine. Each DNA vaccine (pSV1.0-ENV $100 \mathrm{ng}$ per mouse) and adenovirus vaccine (Ad5-ENV $10^{8}$ TCID50 per mouse) were administered into mice via intramuscular injection at 2 -week intervals. Two

FR5 on non-enveloped virus might be conferred at least in part by L-Glu through its activity as a protein antioxidant (Babu and Bawari 1997). In addition to the replicationcompetent adenovirus vectored vaccine such as Ad5 used in this study, the replication-incompetent adenovirus vectors are also commonly used in vaccine development to avoid strong and lasting immune response caused by replication-competent vector (Lapuente et al. 2018). Given that the replication-incompetent adenovirus vectors were generally constructed by deletion of genes involved in viral replication and proliferation such as E1,E3 without changing structure-related genes, whether the vaccine efficacies of these vectors can also be improved by the freeze-drying protectant will be of interest for future studies to assess.

Many studies on vaccine lyophilization focus on vaccine morphology characteristics or use thermography to prove the combination of vaccines and ingredients (Shokri et al. 2019; Yusuf et al. 2019). The number of solutions was determined for prolonged storage at $4{ }^{\circ} \mathrm{C}$. Jae U. Jung found a stable and safe formula that can protect the weeks after the last vaccination, we detected the immunogenicity of the viral vaccines. B-C The protection of antigen-specific $\mathrm{T}$ cell responses was detected by ELISPOT $(\mathbf{B})$ and intracellular cytokine staining $(\mathbf{C})$. Mouse splenocytes were stimulated by the ENV peptide pool. D The protection of specific binding antibodies was measured by ELISA at the same time. Data are the mean of each cohort $(N=6)$, and error bars indicate $\mathrm{SD}(* * * P<0.001, * P<0.05)$.

antigenicity of the Sabin inactivated poliovirus vaccine for 4 weeks at an ambient temperature (Shin et al. 2018). J. Drew found a system that can keep the adenovirus titer at $10^{5}$ (PFU/mL) at ambient temperature (Stewart et al. 2014). Many studies have only explored the protection of viral vaccine infectivity while ignoring the protection of vaccine immunogenicity, which is another important part of vaccine stability. The formulations in our work successfully increased two aspects of vaccine stability and maintained them at a high level (keeping the adenovirus titer above $10^{8} \mathrm{TCID} 50 / \mathrm{mL}$ ); these formulations not only maintained vaccine activity but also protected the vaccine antigenicity from being damaged.

Currently, the storage and transportation of antibodies, proteins and nucleic acids remains a large challenge. It also remains undetermined if our freeze-drying formulas can protect other vaccines, such as poliovirus, rubella virus, and influenza vaccines or parts of vaccine-like liposomes, proteins, and antigens. However, a formulation to protect all aspects of viral vaccine activity remains to be developed. 
Acknowledgements This work was supported by the National Key R\&D Program (2016YFC1303402), the National 13th Five-Year Grand Program on Key Infectious Disease Control (2018ZX10301403, 2017ZX10202102-006), and the Intramural Funding from Shanghai Public Health Clinical Center.

Author Contributions WY, JX and XZ designed the experiments and drafted the manuscript. WY contributed reagents for the formulations. YC, QL and YZ detected the titers of the poxvirus and adenovirus vaccines. $\mathrm{YC}$ and TC performed the animal vaccination and challenge experiments. $\mathrm{YC}$ analyzed the antibody responses and specific $\mathrm{CD}^{+} \mathrm{T}$ cell responses. WY, JX and $\mathrm{XZ}$ supervised all experiments and finalized the manuscript.

\section{Compliance with Ethical Standards}

Conflict of interest The authors declare no competing interests.

Animal and Human Rights Statement Animal care and experiments were conducted with a protocol that was strictly reviewed and approved by the Institutional Animal Care and Use Committee (IACUC) of Shanghai Public Health Clinical Center (Permit Number: 2013-E013). All experiments were performed at least two times with similar results, and one representative result is shown.

\section{References}

Abdul-Fattah AM, Truong-Le V, Yee L, Pan E, Ao Y, Kalonia DS, Pikal MJ (2007) Drying-induced variations in physico-chemical properties of amorphous pharmaceuticals and their impact on Stability II: stability of a vaccine. Pharm Res $24: 715-727$

Babu GN, Bawari M (1997) Single microinjection of L-glutamate induces oxidative stress in discrete regions of rat brain. Biochem Mol Biol Int 43:1207-1217

Baron MD, Iqbal M, Nair V (2018) Recent advances in viral vectors in veterinary vaccinology. Curr Opin Virol 29:1-7

Basu A, Domb AJ (2018) Recent advances in polyanhydride based biomaterials. Adv Mater 30:e1706815

Borde A, Larsson A, Holmgren J, Nygren E (2011) Preparation and evaluation of a freeze-dried oral killed cholera vaccine formulation. Eur J Pharm Biopharm 79:508-518

Briggs H, Ilett S (1993) Weak link in vaccine cold chain. BMJ 306:557-558

Chen C, Han D, Cai C, Tang X (2010) An overview of liposome lyophilization and its future potential. J Control Release 142:299-311

Chen S et al (2012) Investigation on formulation and preparation of adenovirus encoding human endostatin lyophilized powders. Int J Pharm 427:145-152

Hansen LJJ, Daoussi R, Vervaet C, Remon JP, De Beer TRM (2015) Freeze-drying of live virus vaccines: a review. Vaccine 33:5507-5519

Jang YH, Seong BL (2013) Cross-protective immune responses elicited by live attenuated influenza vaccines. Yonsei Med J 54:271-282

Kasper JC, Friess W (2011) The freezing step in lyophilization: physico-chemical fundamentals, freezing methods and consequences on process performance and quality attributes of biopharmaceuticals. Eur J Pharm Biopharm 78:248-263
Kraan H, van Herpen P, Kersten G, Amorij JP (2014) Development of thermostable lyophilized inactivated polio vaccine. Pharm Res 31:2618-2629

Lapuente D, Ruzsics Z, Thirion C, Tenbusch M (2018) Evaluation of adenovirus $19 \mathrm{a}$ as a novel vector for mucosal vaccination against influenza A viruses. Vaccine 36:2712-2720

Madan M, Sikriwal D, Sharma G, Shukla N, Mandyal AK, Kale S, Gill D (2018) Rational design of heat stable lyophilized rotavirus vaccine formulations. Hum Vaccin Immunother 14:2132-2141

Melief CJ, van Hall T, Arens R, Ossendorp F, van der Burg SH (2015) Therapeutic cancer vaccines. J Clin Investig 125:3401-3412

Meri S, Jordens M, Jarva H (2008) Microbial complement inhibitors as vaccines. Vaccine 26(Suppl 8):I113-I117

Mody KT, Mahony D, Cavallaro AS, Stahr F, Qiao SZ, Mahony TJ, Mitter N (2014) Freeze-drying of ovalbumin loaded mesoporous silica nanoparticle vaccine formulation increases antigen stability under ambient conditions. Int J Pharm 465:325-332

Ohtake S, Martin RA, Saxena A, Lechuga Ballesteros D, Santiago AE, Barry EM, Le Truong V (2011) Formulation and stabilization of francisella tularensis live vaccine strain. J Pharm Sci-US 100:3076-3087

Orr MT et al (2014) Elimination of the cold-chain dependence of a nanoemulsion adjuvanted vaccine against tuberculosis by lyophilization. J Control Release 177:20-26

Peetermans J (1996) Factors affecting the stability of viral vaccines. Dev Biol Stand 87:97-101

Prabhu M, Bhanuprakash V, Venkatesan G, Yogisharadhya R, Bora DP, Balamurugan V (2014) Evaluation of stability of live attenuated camelpox vaccine stabilized with different stabilizers and reconstituted with various diluents. Biologicals 42:169-175

Qiu S et al (2014) Fusion-expressed CTB improves both systemic and mucosal T-cell responses elicited by an intranasal DNA priming/ intramuscular recombinant vaccinia boosting regimen J. Immunol Res 2014:308732

Reid MC et al (2019) Models to predict the public health impact of vaccine resistance: a systematic review. Vaccine 37:4886-4895

Shin WJ, Hara D, Gbormittah F, Chang H, Chang BS, Jung JU (2018) Development of thermostable lyophilized sabin inactivated poliovirus vaccine. Mbio 9:e02287-18

Shokri S, Shahkarami MK, Shafyi A, Mohammadi A, Esna-Ashari F, Hamta A (2019) Evaluation of the thermal stability of liveattenuated Rubella vaccine (Takahashi strain) formulated and lyophilized in different stabilizers. J Virol Methods 264:18-22

Siow CR, Wan SHP, Chan LW (2016) Application of freeze-drying in the development of oral drug delivery systems. Expert Opin Drug Deliv 13:1595-1608

Stewart M, Ward SJ, Drew J (2014) Use of adenovirus as a model system to illustrate a simple method using standard equipment and inexpensive excipients to remove live virus dependence on the cold-chain. Vaccine 32:2931-2938

Walters RH, Bhatnagar B, Tchessalov S, Izutsu KI, Tsumoto K, Ohtake S (2014) Next generation drying technologies for pharmaceutical applications. J Pharm Sci 103:2673-2695

Yusuf H, Nugraheni RW, Setyawan D (2019) Effect of cellulose derivative matrix and oligosaccharide on the solid state and physical characteristics of dimethyldioctadecylammoniumliposomes for vaccine. Res Pharm Sci 14:1-11

Zheng Y, Lee PW, Wang C, Thomas LD, Stewart PL, Steinmetz NF, Pokorski JK (2019) Freeze-drying to produce efficacious CPMV virus-like particles. Nano Lett 19:2099-2105 